\title{
HYDROCEPHALUS ASSOCIATED WITH OBLITERATION OF THE LONGITUDINAL SINUS
}

\author{
BY
}

\author{
J. L. EMERY and R. B. ZACHARY \\ From The Children's Hospital, Sheffield
}

(RECEIVED FOR PUBLICATION APRIL 16, 1956)

There are theoretically three possible causes of hydrocephalus; first, an excessive production of cerebrospinal fluid, secondly a block in its circulation and thirdly a defect in reabsorption of the fluid. The majority of cases belong to the second group, that is obstruction to the circulation, and examples of hydrocephalus due to a defect in reabsorption from the subarachnoid space via the pia-arachnoid villi are extremely rare (Potter, 1952; Russell, 1949).

The purpose of this communication is to report two cases, one of a child dying at the age of 2 years in whom hydrocephalus was due apparently to complete organization of the longitudinal sinus over which a bony ridge had developed, and a second child with a history suggesting a cerebral sinus thrombosis, and in whom the development of a similar bony ridge suggested the same aetiology.

\section{Case Reports}

Case 1. This child was three weeks premature. Three weeks after birth he became ill with a temperature of $104^{\circ} F$. and was very sleepy and lethargic. He was given medicine by the family doctor, and appeared to recover from the acute illness, but he still remained for long periods of the day staring in front of him, and the mother thought his eyesight was very poor. At 6 months of age the mother thought his head was large and he was noticed to have a convergent squint. He did make a little progress in general development, but was far below the normal development for the age of 2 at which time he was admitted to hospital.

The head circumference was $24 \frac{3}{4}$ in. and an anterior fontanelle was felt to the right of the midline. Further examination revealed another depression to the left of the midline. He was able to follow a light with his eyes, and would smile and move objects from one hand to the other, but did not say any words with meaning and was not able to drink from a cup.

General physical examination revealed nothing unusual apart from the hydrocephalus. There was no spina bifida.

Ventricular studies showed a symmetrical gross hydrocephalus. The ventricular pressure was more than $400 \mathrm{~mm}$. and the pressure in the lumbar fluid was also more than $400 \mathrm{~mm}$. Three millilitres of phenol red was introduced into the ventricular fluid and appeared in a short time in the lumbar fluid, and the hydrocephalus was thus shown to be communicating.

Following these investigations there was some infection of the cerebrospinal fluid with coagulasenegative Staphylococcus albus, which cleared up with antibiotics. After an unsuccessful attempt to drain the cerebrospinal fluid into the gall bladder an anastomosis was made between the lumbar theca and the left ureter after removal of the left kidney. This procedure was well tolerated and the child went home two weeks after operation, very well and with an obvious depression of the peculiar fontanelle on each side of the midline. The child died quite suddenly a week later.

NeCROPSY. The body was that of a male child, with gross hydrocephalus, but no other external anatomical deformities.

The serous cavities and the abdominal viscera showed no notable abnormality. The appearance of the polythene catheter in the left ureter suggested that there had been some obstruction to the flow of cerebrospinal fluid. The surface of the brain was quite moist, and the convolutions were not abnormally flattened, as is usual in obstructive hydrocephalus. The cortex was approximately $4 \mathrm{~mm}$. thick at the vertex, gradually becoming

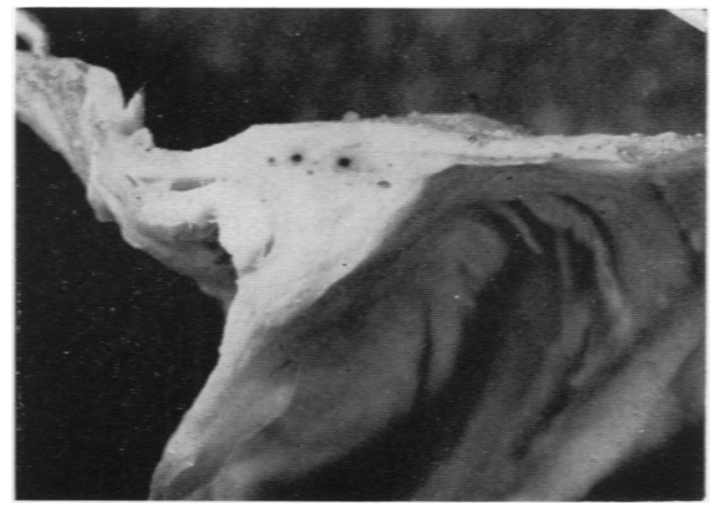

Fig. 1.-View of the falx, longitudinal sinus and attached dura showing the apparent complete obliteration of the sinus. $(\times 1$. 


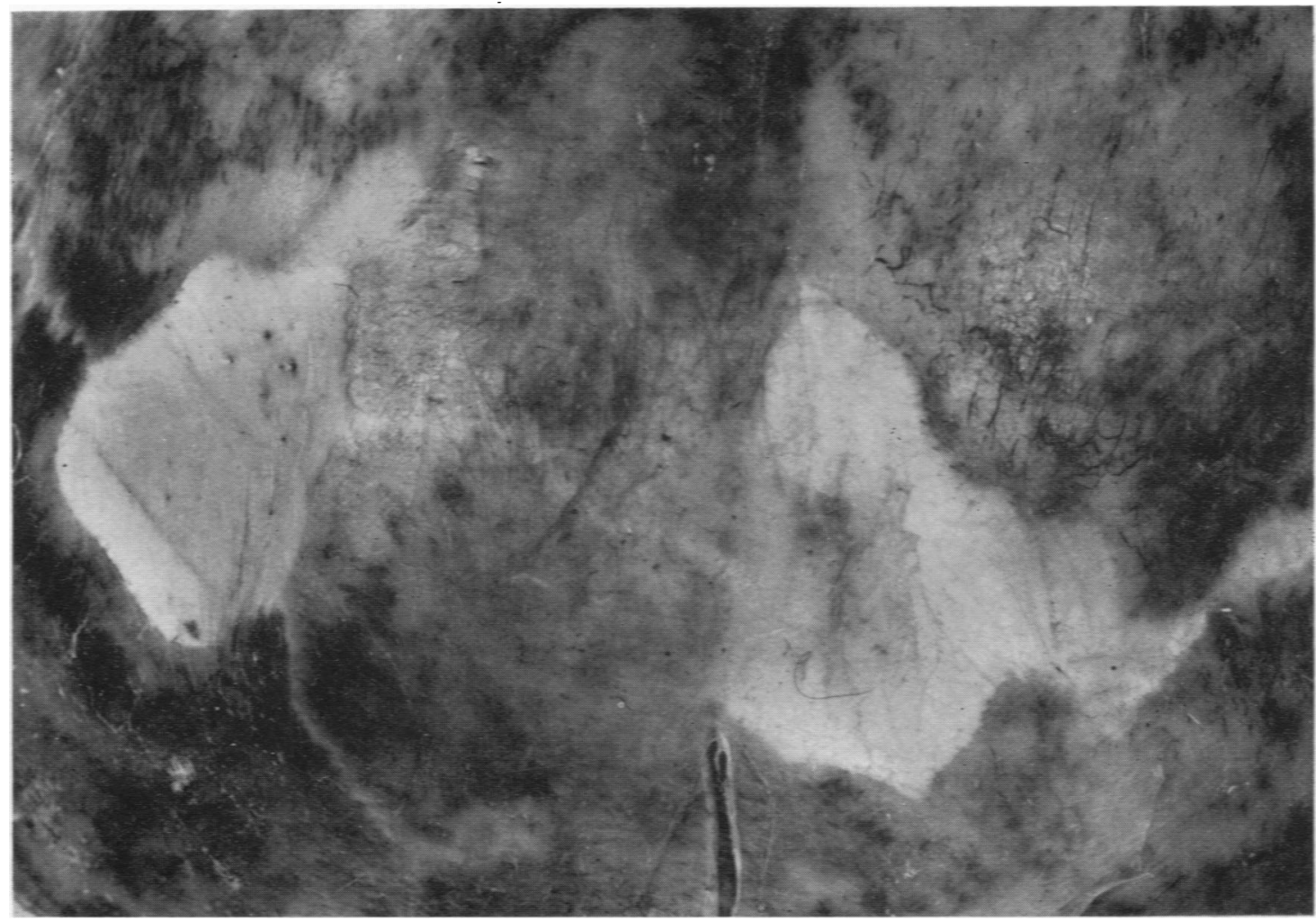

FiG. 2.-View of the outside of the skull at the site of the anterior fontanelle showing the longitudinal bony ridge dividing the normally diamond-shaped space. $\left(\times 1 \frac{1}{2}\right.$.

thicker in the lateral direction and in the region of the occipital poles.

There was a fine granularity over the inner surface of both ventricles, but no brown staining or other evidence of haemorrhage.

The third ventricle was enlarged, and the choroid plexus throughout the lateral ventricles appeared normal. The aqueduct between the third and fourth ventricles was probably within normal limits.

The fourth ventricle appeared to be slightly enlarged.

The cerebellum and medulla showed no Arnold Chiari deformity, and the spinal cord and column were normal. There was no meningocoele or spina bifida.

The longitudinal sinus appeared at first sight to be completely converted into a firm, fibrous mass (Fig. 1), but on closer inspection there were a few small blood vessels within this. The vessels leading into the longitudinal sinus were remarkably small, and there was no evidence of scar tissue or altered blood around them.

The organization of the longitudinal sinus ended abruptly at the tentorium where the sinus was joined by a fairly large blood vessel running in the falx. The straight sinus and lateral sinuses were normal. The skull showed a remarkable thickened, dark, bony ridge immediately over the longitudinal sinus throughout the whole length of the vault. This bony ridge completely divided the anterior fontanelle into two triangular lateral fontanelles (Figs. 2 and 4).

No notable abnormalities were found in the sections from all of the abdominal or thoracic viscera, with the exception of the lungs which had small, long-standing areas of collapse. The longitudinal sinus (Fig. 3) consisted of a dense mass of collagenous material containing several small, thin, strand-like channels of blood vessels lined by a single layer of endothelium. No altered blood pigment was found to suggest thrombosis with canalization. Sections taken from different parts of the longitudinal canal showed a similar picture.

The choroid plexuses appeared normal, and the irregularity of the lining of the ventricles was due to a general undulation of surface, and not to any local disease. No inflammatory changes were seen throughout the ependyma.

The child apparently died as the result of excessive salt loss due to unrestricted drainage of C.S.F. into the urinary tract; we have seen other children who have died in this way.

No gross developmental deformities were found in any of its systems, and the brain, the falx. 


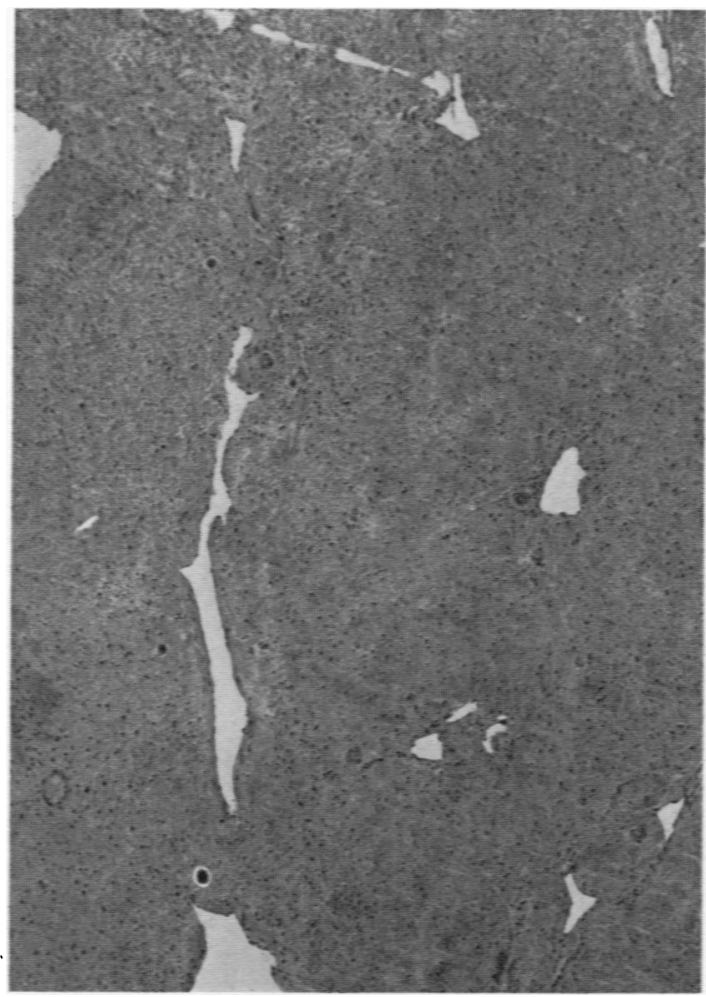

Frg. 3.-Low-power view of the central area of the longitudinal sinus showing dense connective tissue and a few small blood spaces. (Haematoxylin and eosion $\times \mathbf{5 0}$.)

tentorium and foramen magnum suggested that there were no congenital deformities in the brain at the time of birth. The hydrocephalus was clinically of the communicating type, and no obstructive cause for hydrocephalus was found at necropsy; in fact the external appearance of the brain suggested that the brain had been subjected to pressure on both sides instead of the usual inner side only.

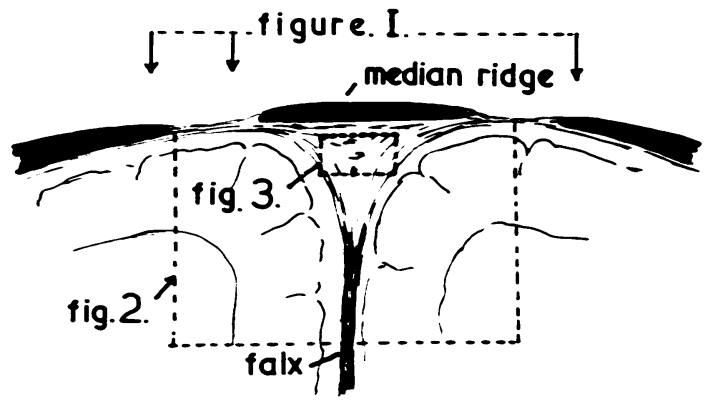

Fig. 4.-Diagram showing the bone ridge and location of the previous figures.
The longitudinal sinus was almost completely replaced by fibrous tissue of very long standing. While the cause of this fibrous replacement was not obvious, if there had been a gross neonatal longitudinal sinus thrombosis it is likely that all of the blood pigments would have been resorbed by this time. Attempts were made to cut sections of vessels from the meninges over the upper surface of the brain, but no pia-arachnoid villi were found.

We feel justified in suggesting that the hydrocephalus in this child was due to a removal of the absorptive mechanism over the cerebral hemispheres by way of the longitudinal sinus. Since the vascularity of the membrane overlying the longitudinal sinus is bound to be intimately related to the sinus, it is difficult to dissociate the overlying bony ridge in the child from the abnormal underlying sinus. It would seem most probable that this abnormal bone production was a direct result of the obliteration of the sinus. The entire clinical picture could well be due to thrombosis preceding the obliteration of the sinus.

Case 2. This baby was a first child, and, apart from toxaemia of pregnancy from about five months, the mother was quite well. The baby was born somewhat postmaturely by forceps delivery. At 1 week of age, he had meningitis due to Bact. coli. This responded well to streptomycin and chloramphenicol, and he appeared to make a complete recovery.

At 3 months of age it was noticed that his head was increasing rapidly in size and at that time was 17 in.

He was admitted to the Children's Hospital, Sheffield, in December, 1953, and was found to have a communicating hydrocephalus in which the ventricles were enormous. The head circumference was $20 \frac{7}{8}$ in. An anastomosis was made between the spinal theca and the left ureter after removal of the left kidney. Drainage of the cerebrospinal fluid appeared to be perfectly satisfactory, and the child was given an additional $3 \mathrm{~g}$. of salt per day to compensate for the loss of electrolytes into the bladder. He was discharged home after 11 days, and appeared to be quite well.

He was again seen three and a half months after the operation. The shunt appeared to be working quite well. The anterior fontanelle was felt to be divided by a bony ridge into two lateral fontanelles. These fontanelles were depressed.

The last time the baby was seen at the Children's Hospital was nine months after operation, when he was 14 months of age and appeared to be making quite good progress. He was still not able to sit up because of the weight of the head, but had started to say words. The head circumference at that time was $23 \frac{3}{8}$ in.

The baby was admitted to another hospital 11 months after operation with a temperature of $103^{\circ} \mathrm{F}$. due to an acute tonsillitis. There was no sign of meningism at that time, but he died within 24 hours. 
The cerebrospinal fluid in the spinal theca was found to be infected, and the cause of death was given as meningitis superimposed upon hydrocephalus.

The pathologist who carried out the necropsy observed the abnormal bony ridge dividing the anterior fontanelle, but did not deliberately examine the cerebral sinuses, or attempt to determine a cause of the hydrocephalus.

The general condition of the child was satisfactory up to the time that he had a severe infection. Following this, hydrocephalus developed, and later the bony ridge overlying the longitudinal sinus in the skull was observed. The child had a communicating type of hydrocephalus which was apparently temporarily relieved by the theca-ureter shunt.

\section{Discussion}

The earliest description of hydrocephalus associated with obstruction of the central venous return known to us is by Browning (1887), who described a child aged 6 years who died with hydrocephalus following an illness lasting six months. In this child there was thrombosis of the straight sinus, and perisinusitis of the longitudinal sinus with apparent obliteration of the smaller vessels leading to the longitudinal sinus. Ellis (1937) recorded a child who developed hydrocephalus following a phase of semi-consciousness. He was able to demonstrate by means of a venogram that there was a complete obstruction to the posterior half of the longitudinal sinus. Bailey and Hass (1937) described two children, both of whom, after a period of normal growth, had convulsions, one at the age of 6 months and the other at 1 month. These children died, and at necropsy both showed longstanding thrombotic occlusion of the longitudinal sinus. Symonds (1937) and Russell (1949) refer to other suggestive, but not conclusive cases. Byers and Hass (1933) in a survey of 50 cases of intracranial venous thrombosis in children found hydrocephalus in eight of these. They state that the hydrocephalus had apparently preceded the thrombosis in five infants, but made no definite statements concerning the other three.

Discussion on the role of thrombosis in the production of hydrocephalus has largely centred around the conditions called 'otitic hydrocephalus'. Whereas some (Symonds, 1937) consider this condition to be a manifestation of cerebral sinus thrombosis, others (McAlpine, 1952) consider the condition to be due to an increased production of cerebrospinal fluid.

Thrombosis of the dural sinuses can produce bizarre features in adults. Ray and Dunbar (1951) describe four cases of 'pseudotumour cerebri', in which a block was demonstrated by venogram, and in one case they removed the clot in the posterior third of the longitudinal sinus, with apparent complete recovery of the patient. Martin (1941) and Martin and Sheehan (1941) reported a series of cases of cerebral sinus thrombosis occurring during the puerperium and causing increased intracranial pressure. Barr and Daws (1956) reported a tumour that apparently produced an increase in intracranial pressure due to obstructing the saggital sinus.

It has not been possible to produce hydrocephalus in experimental animals by occluding the cerebral sinuses. Bedford (1934) tied the great vein of Gaken in the dog and found that there was rapid formation of collateral circulation, and Beck and Russell (1946) failed similarly with ligatures of the longitudinal sinus. However, the venous drainage in experimental animals is not identical with that in man, and the evidence obtained from animals is not conclusive. Surgical approach to the brain occasionally necessitates ligature of the longitudinal sinus. When the sinus is already partly occluded by tumour ligature apparently produces no ill effects (Dandy, 1940), but when no previous occlusion has taken place it seems that the sinus can only be ligatured with impunity anterior to the anastomotic Rolandic veins. If the whole vein is occluded, and the occlusion spreads to the cortical veins, extensive degeneration of the brain ensues (Jaeger, 1942). We have ourselves observed a very great variation in the anastomotic veins in infants' skulls. There are often large vessels running in the falx from the longitudinal sinus to the straight sinus, so that it is to be expected that the effects of occlusion of the longitudinal sinus are bound to vary much between individuals.

In our present Case 1, the whole of the length of the longitudinal sinus was occluded, but not the lateral sinuses or straight sinus. Anastomotic vessels were not seen, and the superficial vessels over the vertex of the brain appeared to be bloodless. No evidence of recent reaction was seen in the sinus nor evidence of old haemorrhage, and the sinus occlusion must have occurred in the distant past, possibly even in utero. In Case 2, we have considerably less evidence than in the other. There is a history of a cerebral incident with clinical meningitis during the newborn period. Cerebral venous thrombosis is frequent in such infants, and occlusion of the sinus could well have occurred at that time. We have never seen the longitudinal bony ridge over the anterior fontanelle in infants in whom hydrocephalus was due to inflammatory scarring around the base of the brain. 
The presence of the longitudinal ridge dividing the anterior fontanelle (Fig. 2) was a striking feature of these cases, and we have not found any record of its occurrence with or without sinus occlusion. Since the venous blood from the dura and periosteal layers overlying the longitudinal sinus flow into the lateral sinus, it would seem justifiable to assume that the calcification is a direct result of the local diminution in the blood flow, due to the obstruction of the sinus.

\section{Summary}

Two children with hydrocephalus of the communicating type are described. In both, an abnormal bony ridge was present, covering the longitudinal sinus and dividing the anterior fontanelle into two parts. At necropsy, the longitudinal sinus in one case was almost completely occluded by dense con- nective tissue. It is suggested that the hydrocephalus in these children was due to a defect in resorption as a consequence of longitudinal sinus thrombosis, and that the bony ridge in the skull was also secondary to the sinus thrombosis.

REFERENCES

Bailey, O. T. and Hass, G. M. (1937). Brain, 60, 293.

Barr, J. and Daws, A. (1956). Brit. J. Surg., 43. 372.

Beck, D. J. K. and Russell, D. S. (1946). J. Neurosurg., 3, 377 Bedford, T. H. B. (1934). Brain, 57, 1 .

Browning. W. (1887). J. Nerv. ment. Dis., 14, 260.

Byers, R. K. and Hass, G. M. (1933). Amer. J. Dis. Child., 45,

Dandy, W. E. (1940). Arch. Surg. (Chicago), 41.244.

Ellis, R. W. B. (1937). Proc. roy. Soc. Med., 30, 768.

Jaeger, J. R. (1942). Arch. Neurol. Psychiat. (Chicago), 48.977

Martin, J. P. (1941). Brit. med. J.. 2. 537.

Martin, J. P. (1941). Brit. med. J., 2. 537.

McAlpine, D. (1952). Arch. Middx. Hosp., 2. 149.

Potter, E. L. (1952). Pathology of the Fetus and the Newborn, p. 422. Chicago.

Ray, B. S. and Dunbar, H. S. (1951). Ann. Surg., 134. 376.

Russell. D. S. (1949). Observations on the Pathology of Hydrocephalus. Med. Res. Coun. Spec. Rep. Ser. (Lond.), No. 265. p. 86.

Symonds, C. P. (1937). Brain. 60, 531. 